\title{
Fenomenología-hermenéutica de la investigación formativa. El formador de formadores: de la imposición a la transformación
}

\section{Phenomenology-herménutica of formative research. The trainer of trainers: from imposition to transformation}

\author{
Alberto Jesús Iriarte-Pupo'
}

Recibido: agosto 15 de 2019

Aceptado: diciembre 20 de 2019

\section{Resumen}

El objetivo de la indagación fue el develar el significado que los maestros en formación, pertenecientes a tres Escuelas Normales Superiores (ENS), de Sucre, Colombia, confieren a la investigación formativa. El abordaje teórico se instaura desde una postura basada en la fenomenología-hermenéutica. Las técnicas de recolección de información fueron: entrevista en profundidad, descripción de la experiencia vivida y observación participante. La muestra fue intencional, con diez estudiantes de últimos semestres. En los resultados se identificaron dos subtemas: Lo paradójico y extraño, y del formador transformativo. En el primero se presentan narrativas sobre tres procesos vivenciales, relacionados con: el cambio de docente, el cambio de la idea inicial de investigación (por imposición) y la percepción de un formador como extraño o raro. En el segundo, se describen experiencias con formadores que promueven la autonomía, la actitud de indagación y la libertad de tomar decisiones. En conclusión, los participantes del estudio conciben la investigación formativa, como: un proceso sistemático y jerárquico, en el que se pueden indagar condiciones relativas a la educación, la pedagogía y la didáctica.

Palabras clave: investigación formativa, fenomenología-hermenéutica, escuela normal superior, formador de formadores.

\begin{abstract}
The aim of the study was to reveal the meaning that teachers in training belonging to three Escuelas Nacionales Superiores (ENS), in the state of Sucre, Colombia; confer to formative research. The theoretical approach is established on the basis of phenomenology-hermeneutics. The techniques of collecting information were: in-depth interview, lived experience description and participant observation. The sample was intentional with ten students from the last semesters. According to the results, two subthemes were identified: the paradoxical and strange and the transformative trainer. In the first one, narratives are presented about three experiential processes related to teacher change, change of the initial idea of the research (by imposition) and the perception of a trainer as a strange or rare. In the second, experiences are described with trainers that promote autonomy, the inquiry attitude and the freedom to make decisions. In conclusion, the study participants conceive formative research as a systematic and hierarchical process, in which conditions related to education, pedagogy and didactics can be investigated.
\end{abstract}

Keywords: formative research, phenomenology-hermeneutics, higher normal school, trainer of trainers.

1 Licenciado en Matemática y Física, Magíster en Educación, Universidad de Sucre, Sincelejo, Colombia. E-mail: albertoiriarte4@yahoo.es ORCID: 0000-0002-4631-6612 


\section{Introducción}

Las nuevas realidades trastocan a todos los puntos de nuestra esfera social, de modo que la educación y la formación se encuentran vinculadas a este cambio de sociedad, desde los aspectos fundamentales de producción, circulación y apropiación cultural (Castell, 2015). Estas mutaciones han originado diferentes crisis, puntos de ruptura y de quiebre, en los disímiles procesos que permean la formación de maestros (Shukshina, Gorshenina, Buyanova \& Neyasova, 2016).

En Colombia, una de las instituciones formadoras de maestros que ha influido notablemente en esta labor, es la denominada Escuela Normal, que a pesar del pasar del tiempo y de diferentes procesos de reforma, sigue cumpliendo con la tarea encomendada desde el año 1821 (Muñoz, 2018). Los problemas que subyacen en la formación inicial de maestros, han sido estudiados ampliamente en las últimas cinco décadas (Amankwah, Oti-Agyen \& Kwame, 2017). Asimismo, las exigencias de la sociedad actual, requieren que los maestros en su formación inicial, adquieran habilidades o competencias investigativas (Zabalza, 2013).

Ahora bien, existen diferentes obstáculos que se oponen al cumplimiento de la tesis del maestro investigador, tales como: debilidad escritural, el temor a la autocrítica y la crítica, dificultad de generar ideas originales, junto con el impacto social del cambio docente (Restrepo, 2008). Esto se une a: la desarticulación entre la teoría y la práctica; pocos espacios y momentos para realizar desde los maestros formadores procesos investigativos; espacios limitados para socializar y reportar los resultados del ejercicio investigativo llevados a cabo por los maestros en formación (Ortiz \& Suárez, 2009). Al igual que la falta de apropiación y sostenibilidad en el tiempo de los aprendizajes obtenidos, en cuanto a la investigación formativa se refiere (López-de Parra, Prada-Arias \& Marín-Arango, 2019). Así también, se exhibe una desarticulación constante entre la formación investigativa y la investigación formativa (Muñoz, 2018).

Lo anterior conllevó a plantear como objetivo del presente estudio: develar el sentido que los maestros en formación, de las Escuelas Normales del departamento de Sucre (Colombia), confieren a la investigación formativa. El abordaje teórico y metodológico de la presente investigación, se construye desde una perspectiva cualitativa y desde una postura teórica basada en la fenomenología-hermenéutica (Van Manen, 2016). El análisis de la información fue realizado por medio del Interpretative Phenomenological Analisys (IPA) (Larkin \& Thompson, 2012), complementado con los métodos reflexivos para develar el sentido de los textos (Van Manen, 2016). En este escrito se da a conocer lo referente a uno de los seis temas analizados, denominado: "El formador de formadores: de la imposición a la transformación". A continuación, se describen diferentes experiencias de vida, narradas por los participantes, que dan cuenta de las transformaciones que se vienen dando en las Escuelas Normales Superiores del departamento de Sucre, Colombia, en cuanto a los procesos de formación de maestros, específicamente en lo que respecta a la investigación formativa.

\section{Marco teórico y metodología}

\subsection{Formación inicial de maestros}

En el presente apartado, se da cuenta de diferentes aspectos ontológicos y metódicos, que se han venido gestando en cuanto al proceso de formación inicial de maestros. Es de aclarar, que uno de los aportes relevantes a la constitución de la tesis del maestro investigador, inició con la propuesta de Dewey (1933), quien planteó un movimiento transformativo del cuerpo magisterial, con el fin de cambiar los modelos basados en una educación asumida para el educando, desde el control, la recepción y repetición de lo dicho por los otros. Desde ahí, se da inicio a proposiciones varias, en diferentes contextos geográficos y temporales, en las que se destacan: el docente "práctico reflexivo" 
(Schön, 1983) y el "profesor reflexivo" (Elliott, 1993). Asimismo, articulada con los postulados de Kurt Lewin (1946), fue evolucionando la tendencia denominada teoría crítica de la educación.

Ahora bien, se identifican junto con el estudio realizado por Gómez (1994), cuatro perspectivas básicas en la formación de maestros. Para el presente trabajo, cobran relevancia dos de ellas, la perspectiva práctica y la perspectiva de reconstrucción social, toda vez que en ambas se invita a formar un maestro investigador (Ossa-Montoya, 2015). Se destaca también que, en la formación de maestros, desde la década de los años 90, ha venido tomando fuerza el enfoque basado en competencias (Zabalza, 2013; Seckel, 2015). Sobre este aspecto, y en atención al presente estudio, se profundizó en lo que respecta a la competencia investigativa, la cual según Delgado (2012), se puede comprender como: la unificación dinámica del saber y el saber hacer con los recursos intelectuales, motivacionales, actitudinales, valorativos de los individuos, en función de un comportamiento investigativo exitoso.

Por otra parte, se identificó la perspectiva trazada por Shulman (1986), referente a la formación de maestros, en cuanto al conocimiento base para la enseñanza, destacándose una prolífera línea de investigación (Tamayo, Flórez \& Velásquez, 2014; Vergara \& Cofre, 2014; Sánchez \& Labarrere, 2015). Así también, se da cuenta de investigaciones que apuntan a mejorar la escisión existente entre teoría y práctica en la formación inicial de maestros (Earl, 2016).

La formación inicial de maestros, está referida a una función determinada ejercida por instituciones específicas, que para el caso que nos convoca, son las Escuelas Normales Superiores de Colombia. Estas cuentan con personal idóneo y especializado, guiados por un currículo que constituye las tareas secuenciales que se operacionalizan mediante el plan de estudios. La formación inicial de maestros, "se encuentra indisolublemente vinculada al desarrollo curricular, en consecuencia al modelo de escuela, de enseñanza, de profesor que se quiere formar y del modelo de prácticas a seguir" (Sayago, 2002).

La formación inicial, es considerada, por tanto, como un recurso concluyente e imprescindible para llevar a cabo demandas y mejoras socialmente solicitadas, tanto en los ámbitos escolares, como en general en lo educativo. Diversos autores sostienen, de manera explícita algunos, y otros de manera implícita, que la formación inicial posee un valor intrínseco cargado de un mundo de significados, experiencias para el aprendizaje que permiten al futuro maestro ir construyendo y reconstruyendo el sentido de la profesión, sus propósitos y orientaciones (Muñoz, 2018).

\subsection{Investigación formativa}

La investigación formativa, según Parra (2004), se define como una herramienta del proceso de enseñanza aprendizaje, es decir la enseñanza a través de la investigación o enseñar usando los diferentes métodos de aproximación a la ciencia, para explicar y comprender fenómenos, y aplicarlos a modelos conceptuales que fundamentan la calidad de los profesionales en formación. El autor expresa, siguiendo la línea de la investigación formativa, "nos referimos a la investigación como una práctica pedagógica de los profesores, como una estrategia para el desarrollo del currículo: un tipo de investigación que tiene una finalidad didáctica (difundir conocimientos existentes), más que epistémica (generar conocimientos nuevos)" (Parra, 2004). En este sentido, Restrepo (2008), esboza que la investigación formativa es formar en “investigación y para la investigación, desde actividades investigativas que incorporan la lógica de la investigación y aplican métodos de investigación, pero que no implican necesariamente el desarrollo de proyectos de investigación completos ni el hallazgo de conocimiento nuevo y universal".

De este modo, la investigación formativa se puede entender, como: una primera e ineludible manifestación de existencia de la cultura de la 
investigación, donde se intenta ayudar al estudiante a buscar y construir conocimientos de una manera metódica y sistemática, aprovechando el propio desenvolvimiento de la búsqueda investigativa (Restrepo, 2008). Un proceso caracterizado por la creatividad del acto, por la innovación de ideas, por los métodos rigurosos utilizados, por la validación y juicio crítico de pares. De esta manera, el conocimiento obtenido, pretende enriquecer la reflexión de la experiencia mediante la deconstrucción y reconstrucción de su enseñanza; y así, la investigación formativa está dirigida a dinamizar la práctica pedagógica reflexiva y transformadora (Arnao, 2015).

\subsection{Sobre la fenomenología y el sentido}

Para entender el sentido desde la propuesta Husserliana, se deben abordar los constructos de Noema y Noesis, que estructuran dicha concepción. Husserl (1976), denomina al acto psicológico individual del pensar Noesis y al contenido objetivo del pensamiento Noema, ambos se encuentran correlacionados en la vivencia intencional. "la correlación se entiende como aquello que permite conocer el objeto real en tanto correlato de sentido. La correlación funda la relación entre la conciencia y su objeto. La correlación es indestructible, se establece entre dos polos inseparables" (Hincapié, 2013). En cuanto al Noema, este "se refiere al contenido en cuanto tal de la vivencia. $Y$ que hay que entenderlo como aquello que es dado, propio de la realidad de la cual hemos hecho experiencia" (ibíd), es necesario entender a su vez, que la realidad objetiva en completitud de perspectivas es un reto inalcanzable. Por tanto, lo donado de los objetos se convierte en posibilidades de estructuración eidética, hacen parte de los diversos modos de ser y de darse del Noema. Por su lado, la Noesis es entendida como la:

(...) pura actividad de la conciencia que le otorga al "contenido" su modalidad de acto consciente en determinada forma, especificando sus características noemáticas, es decir, ya sea en cuanto "percibido", o como "recordado", o en cuanto "deseado", "juzgado", etc., es el acto mismo del percibir, recordar, desear, juzgar, etc. (ibíd., p. 6)

Esta dualidad entre Noema y Noesis, debe entenderse desde el mundo de la vida, desde el Dasein (ser-ahí) de Heidegger, una postura filosófica, ontológica, y epistemológica, que se constituye como una estructura indisoluble en una unidad noético-noemática. Esta relación noético-noemática, se entiende desde la oposición correlacional del Noema (cosa pensada) a la Noesis (acción del pensar). En consecuencia, el sentido desde la perspectiva fenomenológica de Husserl, es "el efecto de la praxis, del movimiento de construcción de la unidad noético-noemática, esto es, que sitúa a un cierto número de actos de la conciencia frente a un cierto número de donaciones de la cosa a la intencionalidad" (Potestá, 2013, p. 28). Es en el telos, en la finalidad, que se induce a la intencionalidad, por lo cual la conciencia situacional se caracteriza por ser un criterio del sentido. Dicho de otra manera, es la intención correlativa entre Noema y Noesis, dada en la conciencia, constituida en un contexto histórico-cultural particular, la que moviliza al sentido; posibilitando a través de la intercomunicación, la significación compartida.

El sentido ocurre en la vivencia, la cual, a su vez, corresponde a un eidos, a una idea, a una esencia. Por consiguiente, donde se instale una propuesta de estudio de los mundos posibles, del mundo subjetivizado, se hace necesario fenomenologizar, volver la mirada hacia el sujeto, deconstruir, genealogizar, lo normalizado. De esta forma la propuesta fenomenológica, más que insistir en una refutación de la metafísica imperante, provee de herramientas que dan la posibilidad de estudiar de manera incesante el sentido (o los sentidos) dado (s) en el mundo en que de manera efectiva vivimos (Vargas-Guillen, 2012).

\subsection{Metodología}

Esta investigación se planteó desde una perspectiva metodológica de tipo cualitativo, con lo cual 
se buscó realizar el estudio de las experiencias vividas de los maestros en formación, así como de la interpretación y descripción de las características particulares de su discurso y del lenguaje corporal, que se logró observar durante las entrevistas y en la observación participante. De esta manera, aproximarse a develar el sentido, respectivo al proceso llevado a cabo en cuanto a formarse como maestros investigadores. La investigación cualitativa parte del supuesto que el mundo social está constituido por medio de significados y símbolos, lo que implica la búsqueda de esta formación y de sus representaciones a través de la experiencia de los sujetos (Hernández, Fernández \& Baptista, 2014).

El tipo de diseño escogido pertenece a la tradición fenomenológica-hermenéutica, que se enfoca en el estudio de las experiencias individuales subjetivas de los participantes. Asimismo, responde a la pregunta ¿Cuál es el significado, estructura y esencia de una experiencia vivida por una persona, grupo o comunidad, respecto de un fenómeno? Los sujetos de estudio fueron los estudiantes del Programa de Formación Complementaria, pertenecientes a las Escuelas Normales Superiores (ENS), del departamento de Sucre, así: Institución Educativa Normal Superior de Sincelejo, Institución Educativa Normal Superior de Corozal y La Institución Educativa Normal Superior de Majagual. La muestra fue intencional, con una participación total de diez estudiantes de últimos semestres (IV o V): seis de sexo masculino y cuatro de sexo femenino.

La recolección de los datos se efectuó utilizando una entrevista en profundidad semiestructurada, la cual fue validada (contenido), por juicio de expertos. Así también, se utilizó la descripción de la experiencia vivida y la observación participante, donde se registraron los datos por medio de un texto descriptivo y anecdótico, y un diario de campo. El análisis de los datos implicó usar el Interpretative Phenomenological Analisys (IPA) (Larkin \& Thompson, 2012) conjuntamente con el enfoque hermenéutico planteado por Van Manen
(2016). Las convenciones utilizadas fueron: EP1, Donde E significa entrevista, y P1 participante número uno; DEVP1, DEV significa descripción de la experiencia vivida, P1 participante número uno.

\section{Resultados y discusión}

Con el objetivo de comprender el sentido que le dan los maestros en formación a la investigación formativa, se presenta a continuación uno de los temas, con sus respectivos subtemas, que emergió del proceso de análisis de datos, denominado: "el formador de formadores: de la imposición a la transformación". Como apunta Aguas (2014), los temas surgen de las categorías finales y los subtemas de la base de la consideración conceptual previa.

Todos los participantes se refirieron, de una forma u otra, a la relación existente entre sus formadores (sean estos docentes titulares de la ENS o docentes tutores en las escuelas de práctica), y ellos como estudiantes. Se entiende que el formador de formadores es un mediador del aprendizaje, a quien le corresponde "mostrar coherencia entre discurso y práctica, debe asumir personalmente los valores que pretende transmitir; debe vivir el compromiso con la profesión de la misma forma que espera que los formandos lo asuman cuando se conviertan, a su vez, en formadores" (Vaillant \& Marcelo, 2001). En el contexto particular de la investigación formativa, estos componentes son tanto teóricos como prácticos, los cuales se deben articular en la medida en que se desarrollan las competencias profesionales. Lo que sigue es la forma en que varios participantes rememoraron algunos aspectos referentes a este tema, a lo largo del proceso de su formación. Se pueden identificar dos categorías esenciales en particular: la primera denominada "lo paradójico y extraño", y la segunda llamada "del formador transformativo".

\subsection{Lo paradójico y extraño}

En este apartado se deconstruyen las narraciones de los participantes, en cuanto a los aspectos en 
los cuales sienten alteración del deber ser con lo que viven. Es decir, aquellos momentos de la formación, en que los estudiantes perciben y advierten, que deberían ser de otro modo; sin embargo, por diferentes motivos y acciones de aquellos que los están formando, se convierten en normalizaciones.

La experiencia vivida por P1 se relaciona con la transformación de una idea inicial propia para investigar, la cual debió ceder por distintas situaciones que se detallan en su narrativa:

“Entonces..., me pareció un problema interesante, apareció un proyecto al instante y motivador para mí misma como una experiencia propia, (...), algo que me ayudaría a mí a seguir adelante en el proceso de investigación. Pero luego con... este..., con la llegada de la nueva docente, (...), se cambiaban las cosas, porque hubo una nueva idea de formar parte de un grupo grande para trabajar en un proyecto llamado (...). Entonces me sentí como un poco sola (...), sentía la frustración de que no iba a seguir trabajando con lo que yo había empezado, y quería realizar". (EP1)

De igual manera, la experiencia narrada por P2, evidencia la renuncia de su motivación inicial, por argumentos o acciones realizadas por el formador de maestros. P2 comenta que:

"Como nosotros, para el proceso de la práctica tenemos que desplazarnos a la zona rural, entonces yo llegué a una escuelita ahí, y observé unas necesidades, y le dije a mis compañeros..., podemos hacer una investigación, sobre la falta de recursos en la escuela. Y al llegar acá, a la escuela (a la Normal Superior), esteee..., la seño de investigación nos dice: "no, estooo..., eso no se puede investigar", eso sacó unas conclusiones que me dejó fuera y yo quería llevar a cabo esa investigación, allá en esa escuelita".

"Yo principalmente dije: "ombe..., la seño qué va a saber de investigación ni que nada, eso se puede investigar, yo le voy a preguntar a..." y mis compañeros me decían: ¡cállate, no te pongas a pelear con esa seño, porque después el que va a llevar la de perder eres tú!, pero yo decía... ¡yo quiero la escuela, yo quiero! ..., y eso fue lo que sucedió... Es un sueño frustrado (...). (Me dije), qué hago aquí, yo debiera salirme; hasta sentí que esto no era lo mío, porque yo quería ayudar a la escuelita, quería ayudarles, pero me sentí decepcionado, me sentí como..., no tuve para refutarle eso a la seño, entonces me quedé callado, me quedé en blanco y... sentía como si la sangre se me hubiera ido acá, a la cabeza, me sentía la cabeza grande y no sé..., las orejas se me calentaron. Me quemaba. Yo me sentí algo fastidioso, yo no quería estar en ese momento ahí... Me sentí que ese tiempo, fue lo más largo de la clase, es una hora que yo la sentí como..., como de 80 minutos, y larguísimo se me fue el tiempo, yo quería irme para mi casa rápido y nada..., dónde se acababa la hora... eso me llevó quizás a tomarle fastidio a la investigación; nombe..., qué voy a hacer yo con eso, si no puedo ayudar a otros". (EP2)

En este relato en particular, se pueden distinguir lo que Van Manen (2016) denomina existenciales: la relación vivida (la relacionalidad); el cuerpo vivido (la corporalidad); el espacio vivido (la espacialidad); el tiempo vivido (la temporalidad); y las cosas y tecnologías vividas (la materialidad). Como expresa el autor: "las nociones de relación, cuerpo, espacio, tiempo y cosas vividas son existenciales en el sentido en que pertenecen al mundo de la vida... Todos vivenciamos nuestro mundo a través de estos existenciales" (p. 349). Por lo tanto, los existenciales son útiles para explorar aspectos significativos del mundo de la vida y de los fenómenos particulares que en ella suceden.

Varios aspectos se relacionan con los sentimientos de frustración, malestar, decaimiento, tristeza, incomodidad, decepción e inconformidad, detallados por los maestros en formación, cuando se realiza un cambio en la motivación, o idea inicial, del proyecto de investigación. En este sentido, se logra evidenciar que el cambio de tutor, jugó un papel fundamental en lo que respecta a la 
alteración de las ideas y motivaciones primeras de los estudiantes. Así lo plantean P1 y P9:

"Pero luego con... este..., con la llegada de la nueva docente, (...), se cambiaban las cosas" (EP1).

"Un docente decía que sí podíamos..., (sin embargo), tuvimos cambios de docentes, con lo cual nos cambió el hilo conductor que llevamos, fue un cambio brusco y tocó volver a empezar prácticamente". (EP9).

Si bien el cambio de tutor o docente, es algo que es posible e inherente a la institucionalidad educativa, la demanda de los estudiantes es en otro sentido. Se evidencia entonces, que la contradicción, la no conversación y el no llegar a acuerdos entre los formadores de formadores, obstaculiza el proceso. Lo anterior, posibilita una violencia discursiva que de manera explícita o implícita, se configura en una degradación de la subjetividad del otro, que para el caso es el maestro en formación. Piénsese en un tipo de violencia que actúa imperceptiblemente sustrayendo las palabras que podrían promover el pensamiento y trascender lo ya pensado y establecido. Con esta sustracción pueden llegar a degradarse no solo los procesos de formación, sino la vida entera de los estudiantes.

En este sentido, si el ideal de la formación es promover el pensamiento crítico (Mendoza, 2015), y a su vez dar respuesta a las necesidades del contexto donde se desenvolverán en un futuro los maestros que se están formando en este momento (Ortiz \& Suárez, 2009; Parra, 2007), este ideal se ve escindido, cuando la imposición y coacción se ponen en práctica por parte del formador de formadores. Es paradójico entonces, que mientras se enaltece en el discurso la formación crítica, en la práctica se sigan imponiendo ideas dogmáticas del maestro como autoridad deontológica.

Desde otras experiencias vividas, sobresalen aspectos que obscurecen la posibilidad de llevar a cabo un proceso de formación en investigación, tal como se propone en la teoría. Desde este punto, vale la pena deconstruir lo descrito por P6:

"Fue un profesor no se..., él era raro, al profesor no se le veía el entusiasmo como tiene que ser un docente, era impuntual, nos decía..., bueno, hagan esto y nosotros (le) preguntábamos a él ¿cómo se hace? y él nos decía, ¡hay no, háganlo.... ya vuelvo!; entonces, el primer encuentro de la investigación como tal..., me preguntaba, en serio..., ¿esto es la investigación?..., no le encontraba sentido, no aprendí nada y no explicaba, me preguntaba..., en serio, ¿esto es la investigación?... ¡Profesor!, pero díganos cómo se hace.... el docente nos decía las cosas para salir del paso". (EP6)

Las expresiones que resaltan en el discurso del participante en cuestión, tales como: rareza; falta de entusiasmo; ausencia personal; impuntualidad; carencia de aprendizaje (que para el caso sería enseñanza); y pobreza de explicación, se convierten en unidades polivalentes e incompatibles, de lo que debe ser un formador de formadores. Es de anotar, que este problema es significativamente diferente, a aquel donde se expresa la existencia de alto grado de asignaturismo y rutinización de los procesos investigativos, que llevan a la pérdida de sentido y significado del proceso (Parra, 2007; Peralta \& Monterroza, 2014). Lo que en cierta forma se expresa, devela que, en la práctica docente, la rareza y la paradoja son posiciones que existen y subsisten, configurándose en hitos extraños que permanecen en los eventos de formación. En consecuencia, se establece entonces un sinsentido.

En este mismo alcance, el estudiante $\mathrm{P} 10$ relata su experiencia vivida diciendo: "los maestros que supuestamente nos deben enseñar a nosotros sobre investigación, ellos tampoco como que saben mucho..., sí, porque yo he visto que a uno no le explican bien, o no les entiende uno bien y termina a veces, más enredado que cuando no sabía nada" (EP10). Se vislumbra entonces, la contradicción: cómo una persona que debe 
inspirar a otros, en cuanto a la importancia y relevancia de llevar a cabo el proceso investigativo como base de la enseñanza, y del ser maestro del presente siglo, se convierte en aquello que obstaculiza, veda, niega el proceso de transformación con su práctica, con el contraejemplo del Ser maestro investigador. Este tipo de posiciones enrarece la labor.

\subsection{Del formador transformativo}

En este apartado se describen e interpretan diferentes narrativas, expresadas por los participantes, en cuanto a la experiencia vivida en correspondencia con la percepción de la relación originada con sus formadores. Una relación que, frente a la hegemónica, plantea la colaboración; frente a la violencia discursiva, el aprender juntos. Una relación que esté orientada más hacia el aprendizaje de los procesos investigativos como apoyo a la transformación de la práctica pedagógica, que a perpetuar posiciones institucionales. Las experiencias relatadas por los maestros en formación, brindan la posibilidad de entender en lo antes expuesto.

"Yo sentía que aprendía más con la maestra y que sentía más satisfacción con respecto a la formación en la investigación, sentía que ella me aportaba mucho más (...) Ella está mucho más al pendiente de cómo estamos trabajando, de si estamos cumpliendo con los compromisos, que si nos estamos reuniendo como grupo en el proyecto de investigación, dependiendo a cuál de los proyectos..., entonces ella lleva como un control de eso y nos sentimos como que..., bueno, respaldados por parte de ella". (EP1)

"Cabe resaltar a las profesoras que fueron piezas fundamentales, que van acompañándote en el proceso, que te motivan y te entusiasman y te dicen "oye..., esto lo estás haciendo bien"o"te falta esto" y en ese proceso, vas enamorándote de cada cosa que haces (...) gracias a Dios nos cambiaron el docente y empezamos con unas maestras muy buenas, excelentes..., las admiro demasiado, y ya uno le va encontrando sentido a todo lo referente a la investigación... Cuando me cambian el profesor, se transforma completamente la mente, el pensamiento". (EP6)

Cuando los maestros en formación encuentran en sus formadores: apoyo, respaldo, disposición, disponibilidad, guía, ayuda, acompañamiento y colaboración, sienten que lo que están realizando tiene sentido. Es decir, conforman un significado subjetivo de la importancia de la investigación en su proceso de formación. Asimismo, las anteriores actitudes promueven el fortalecimiento de las competencias profesionales que los maestros están desarrollando a lo largo de la carrera magisterial.

Se resalta entonces, que la posición del formador de formadores como acompañante y guía, debe promover la autonomía, así como una actitud de indagación en los maestros en formación, que les permita tomar decisiones en pro de transformar su práctica docente. Desde esta consideración, se destaca uno de los principios de la teoría crítica de la educación, en donde se expresa que, la Pedagogía Crítica "forma al docente en una didáctica fundamentada en la investigación-acción deliberativa, colaborativa y autónoma. La que es llevada a cabo en unión con los colegas y sus discentes en lo relacionado con y acerca de su práctica pedagógica diaria" (López, 2010, p. 17).

Por otro lado, es de subrayar que las actitudes percibidas como positivas por los participantes, estimulan en ellos, entre otros aspectos: la motivación, el aprendizaje, la satisfacción, el enamoramiento por la carrera, la admiración por el otro, el sentido sobre la investigación. Condiciones necesarias para seguir formándose y transformándose, de manera constante, en un maestro investigador. En cuanto a la motivación, se puede decir que es el deseo, o el interés por algo, que brota desde dentro del maestro en formación. Es como una fuerza que provoca interés por alguna cosa, que para el caso específico, es el de querer aprender a investigar. Cuando el formador de formadores 
motiva a los estudiantes, traza un camino expedito para configurar un significado positivo sobre el proceso investigativo.

Por su parte, cuando uno de los participantes comenta que sintió satisfacción con respecto a la formación en investigación (P1), debido al cambio de docente, sintiéndose identificado con el proceso de acompañamiento llevado a cabo por el formador, posibilita la comprensión sobre cómo este aspecto influye en su educación. De manera tal que, el aumento de la satisfacción, en lo que respecta a la enseñanza, aportes y respaldo del docente, conlleva a tener una motivación mayor sobre el aprendizaje y desarrollo de las competencias profesionales.

Otras cuestiones que se destacan en los relatos de los maestros en formación, son las referentes al enamoramiento por la carrera y la admiración por el otro. A manera de hipótesis, se supondrá que uno de estos aspectos conlleva al otro. En este caso la admiración por el otro, posibilita la idea de un enamoramiento no del otro, sino de lo que hace, y que complementa el deseo de ser como él. El maestro en formación se identifica con el docente titular, es decir, lo utiliza como sustitución para crecer, para abrirse caminos de saber o de perspectivas. Desde esta posición el enamoramiento aparece, una pasión que se despierta y transforma día a día. Una utopía que sugiere seguir caminando, aprendiendo, re-creándose, para acercarse a lo admirado, a lo amado, a ser un maestro crítico y reflexivo, tal como aquel que deslumbra e impresiona con su quehacer.

Cada posibilidad de transformación, de apoyo, de guía y respaldo, van configurando en los maestros en formación un sentido positivo sobre la investigación. Promoviendo en ellos, la autonomía, la motivación y la vocación por la carrera docente. Lo anterior desde la conformación de una cultura investigativa, en donde se impulsan valores de colaboración y cooperación en pro de generar alternativas de respuesta a las necesidades y requerimientos educativos y pedagógicos de sus contextos (Jaramillo et al, 2007). Es decir, se va constituyendo un formador como intelectual transformativo (Iriarte, 2015).

\section{Conclusiones}

En las descripciones brindadas por los maestros en formación, se lograron identificar, para este tema en particular, dos subtemas: Lo paradójico y extraño, y del formador transformativo. En el primero, se presentan narrativas en cuanto a tres procesos vivenciales que se relacionan con el cambio de docente, el cambio de la idea inicial de investigación (por imposición) y la percepción de un formador como extraño o raro. En el segundo, se describen experiencias con formadores que promueven la autonomía, la actitud de indagación y la libertad de tomar decisiones.

Con respecto al primer subtema, y las categorías emergentes, los participantes expresaron que en estos casos emanan, entre otros, sentimientos de frustración, malestar, tristeza, decepción, inconformidad, incomodidad. Asimismo, describen cómo ponen resistencia a los mecanismos de poder, realizados por los docentes, terminado en algunos casos en dominación, autoritarismo y puesta en marcha de la violencia discursiva. Lo anterior, crea ciertas paradojas tales como: tener un tutor que no realiza tutoría, o un acompañante que no acompaña, conllevando a obstaculizar, vedar el proceso de transformación. Creando, a su vez, un sentido negativo de la investigación formativa.

En cuanto al segundo subtema, los maestros en formación opinaron que cuando un docente realiza su papel de guía, acompañante, colaborador, donde respalda las diferentes iniciativas, ayuda a conseguir las metas y logros trazados, brindando disponibilidad y estando dispuesto a la labor de formar; esto promueve la motivación y el aprendizaje, derivando en sentimientos de admiración y enamoramiento por la investigación y la carrera. En este caso, el docente se convierte en un formador transformativo, el cual contribuye 
a construir una cultura de la investigación, así también un sentido positivo de la misma.

En conclusión, se sintetiza que el sentido constituido sobre investigación formativa, por los participantes del estudio, la conciben como"un proceso sistemáticoy jerárquico, en el que se puede indagar, tanto condiciones endógenas como exógenas, relacionadas con la educación, la pedagogía y la didáctica. Para "ir más allá" de lo normalizado, y de esta manera aportar a la búsqueda de soluciones plausibles de las problematizaciones planteadas, por medio de la innovación y la transformación, tanto de las representaciones mentales como de las actuaciones subjetivas e intersubjetivas, de los implicados en el campo de la formación".

\section{Referencias}

Aguas, P. (2014). A Phenomenological Study of Key Stakeholders' Lived Experiences While Implementing an Aligned Foreign Language Curriculum (Tesis doctoral). University of Phoenix, Arizona, USA.

Amankwah, F., Oti-Agyen, P., \& Kwame, F. (2017). Perception of Pre-Service Teachers' Towards the Teaching Practice Programme in College of Technology Education, University of Education, Winneba. Journal of Education and Practice, 8 (4), 13-20. Recuperado de: https://eric. ed.gov/?id=EJ1133036

Arnao, M. (2015). Investigación formativa y competencia comunicativa en educación superior. (Tesis doctoral). Universidad de Málaga. Málaga, España.

Carr, W., \& Kemmis, S. (1988). Teoría Crítica de la Enseñanza. La investigación acción en la formación del profesorado. Barcelona, España: Ediciones Martínez Roca.

Castell, M. (2015). Networks of outrage and hope. Social Movements in the Internet Age. Reino Unido: Polity Press
Delgado, M. H. (2012). Mejoramiento de la competencia investigativa en la formación de docentes de licenciatura en educación básica. (Tesis doctoral). Universidad Libre Colombia. Bogotá. Colombia.

Dewey, J. (1933). How We Think: A restatement of the relation of reflective thinking to the educative process. Chicago, USA: Henry Regnery.

Earl, K. (2016). Reflective practice and inquirí: Let's Talk more about inqury. Teachers and Curriculum, 16 (2), 47-54. doi: https://doi.org/10.15663/tandc. v16i2.139

Elliott, J. (1993). La investigación-acción en educación. Madrid, España: Morata.

Hernández, R., Fernández, C., \& Baptista, P. (2014). Metodología de la investigación. México: McGraw-Hill.

Hincapié, B. (2013). "Del yo al objeto". La relación noesis noema y sus consiguientes estructuras. Relatoría sobre textos Fundamentales II. Recuperado de: https://profesorvargasguillen.files.wordpress. com/2013/03/relatorc3ada-nc3b3esis-nc3b3ema-11-de-mayo.pdf

Husserl, E. (1976). Investigaciones lógicas. Traducción de Morente, M., \& Gaos, J. Revista de occidente S. A. Madrid, España: Ediciones Castilla S.A.

Iriarte, A. (2015). El maestro como intelectual de la cultura. Revista Escenarios, 17, 6 - 22. Recuperado de: http://revistas.cecar.edu.co/escenarios/article/ view/144/135

Jaramillo, R. et al. (2007). Cultura investigativa y formación de maestros. Medellín, Colombia: Ediciones Litoimpresos y Servicios Ltda.

Larkin, M., \& Thompson, A. (2012). Interpretative phenomenological analysis. En: A Thompson \& D Harper (eds), Qualitative research methods in mental health and psychotherapy: a guide for 
students and practitioners, 99-116. John Wiley \& Sons, Oxford.

Lewin, K. (1946). Action research and minority problems. Journal of Social Issues, 2 (4), 34-46.

López, G. (2010). Apuntes sobre la pedagogía crítica: su emergencia, desarrollo y rol en la posmodernidad. Santiago de Cali, Colombia: Universidad San Buenaventura.

López-de Parra, L., Prada-Arias, E. R., \& MarínArango, D. J. (2019). Representaciones sociales sobre prácticas investigativas. Condiciones en la universidad. Entramado, 15 (1), 192-211. doi: https://dx.doi.org/10.18041/1900-3803/ entramado.1.5405

Mendoza, P. (2015). La investigación y el desarrollo de pensamiento crítico en estudiantes universitarios. (Tesis doctoral). Universidad de Málaga. Málaga, España.

Muñoz, J. (2018). La práctica pedagógica investigativa en las Escuelas Normales Superiores del norte del departamento de Nariño. (Tesis doctoral). Universidad de Nariño. San Juan de Pasto, Colombia.

Ortiz, R., \& Suárez, J. P. (2009). La formación de maestros y la noción maestro investigador (1996 2005) un espacio para la reflexión y el debate. (Tesis de maestría). Universidad de Antioquia. Medellín, Colombia.

Ossa-Montoya, A. (2015). Lo pedagógico y el maestro investigador. Revista Virtual Universidad Católica del Norte, 44, 102-118. Recuperado de: http://revistavirtual.ucn.edu.co/index.php/ RevistaUCN/article/view/618/1153

Parra, M. C. (2004). Apuntes sobre la investigación formativa. Educación y Educadores, 7, 57-77.

Parra, R. (2007). La enseñanza de la investigación en las Escuelas Normales. Dirección de calidad de la educación preescolar, básica y media. Subdirección de mejoramiento. Bogotá: Ministerio de Educación Nacional.

Potestá, A. (2013). El origen del Sentido. Husserl, Heidegger, Derrida. Santiago de Chile, Chile: Ediciones Metales Pesados.

Restrepo, B. (2008). Formación Investigativa e investigación Formativa: Acepciones y Operacionalización de esta última y Contraste con la Investigación Científica en Sentido Estricto. Material didáctico: Diplomado en Investigación. ESUMER. Medellín, Colombia.

Sánchez, L., \& Labarrere, A. F. (2015). Interacción estudiante-investigador. Relación pedagógica y profesional que sustenta la formación en el postgrado. Revista Electrónica Actualidades Investigativas en Educación, 15 (2), 1-18.

Schön, D. (1983). The effective Practitioner: How Professonals Think in action. New York: Basic Books.

Seckel, M. J. (2015). Competencia en análisis didáctico en la formación inicial de profesores de educación general básica con mención en matemática. (Tesis doctoral). Universidad de Barcelona. España.

Shulman, L. (1986). Those who understand: knowledge growth in teaching. Educational Researcher, 15 (2), 4-14.

Shukshina, T., Gorshenina, S., Buyanova, I., \& Neyasova, I. (2016). Practice-Oriented Teachers' Training: Innovative Approach. International Journal of Environmental \& Science Education, 11 (16), 9125-9135. Recuperado de: https:// pdfs.semanticscholar.org/317f/3dc70d625dd8eb1963cc72f58613ef8d0a88.pdf

Tamayo, O., Flórez, G., \& Velásquez, J. (2014). Conocimientos necesarios para la enseñanza: una categoría fundamental en la formación de maestros. Caldas, Colombia: Editorial Universidad del Tolima 
Vaillant, D., \& Marcelo, C. (2001). Las tareas del formador. Málaga, España: Ediciones Aljibe.

Van Manen, M. (2016). Fenomenología de la práctica. Métodos de donación de sentido en la investigación y la escritura fenomenológica. Traducido por Aguirre, J.C., \& Jaramillo, L.G. Popayán, Colombia: Editorial Universidad del Cauca.

Vargas-Guillen, G. (2012). Fenomenología, formación y mundo de la vida. Problemas teóricos y metodológicos de la fenomenología. España: Editorial Académica Española.

Vergara, C., \& Cofré, H. (2014). Conocimiento Pedagógico del Contenido: ¿el paradigma perdido en la formación inicial y continua de profesores en Chile? Estudios Pedagógicos, 40 (1), 323-338. doi: http:// dx.doi.org/10.4067/S0718-07052014000200019

Zabalza, M. A. (2013). Competencias docentes del profesorado universitario. Calidad y desarrollo profesional. Madrid, España: Narcea. 